\title{
Unilateral adrenal infarction in pregnancy with associated acute hypoadrenalism and subsequent spontaneous biochemical and radiological resolution
}

\author{
Najeeb Shah ${ }^{1}$, Harshal Deshmukh ${ }^{1}$, Muhammad Akbar ${ }^{1}$, Shah Malik ${ }^{1}$, Yamna Saeed ${ }^{1}$, and \\ Belinda Allan ${ }^{1}$ \\ ${ }^{1}$ Hull University Teaching Hospitals NHS Trust
}

October 30, 2021

\begin{abstract}
Authors describe a rare case of acute adrenal infarction in pregnancy resulting in hypoadrenalism requiring hydrocortisone replacement therapy and anticoagulation with subsequent resolution of the radiological changes and complete recovery of the glucocorticoid reserve during clinical follow-up.
\end{abstract}

\section{Introduction}

Adrenal infarction is an uncommon condition which is rarely seen in pregnancy. Hypoadrenalism secondary to unilateral adrenal infarction is not expected. We describe a case of a 25 year-old pregnant female who presented with left flank pain and after the initial investigations returned normal, a contrast-enhanced computed tomography (CT) scan of the abdomen and pelvis was done due to persistent symptoms which revealed left adrenal infarction. Day 2 random serum cortisol was $557 \mathrm{nmol} / \mathrm{L}$ (200-700). The patient had spontaneous rupture of membranes, and the baby was delivered by an emergency C-Section. Day 3 morning (0900) serum cortisol was grossly abnormal at 78nmol/L (200-700). The patient was commenced on hydrocortisone and anticoagulated with warfarin. Six months post-event, follow up magnetic resonance imaging (MRI) scan of the adrenal glands revealed normal appearances. Her adrenocortical steroid reserve completely recovered after 16 months at which point she was successfully weaned of hydrocortisone and was discharged from the endocrinology follow-up.

This case is unique as not only does it describe a rare diagnosis, it highlights that unilateral adrenal infarction can lead to adrenal insufficiency and haemodynamic instability. Recognition of adrenal infarction as a cause of abdominal pain during gestation is challenging due to the obscurity of this diagnosis and the fact that during evaluation of such cases, attention usually turns to the usual culprits, for example; physiological (uterine enlargement/increase volume of amniotic fluid), preterm labor, placental abruption, appendicitis, cholecystitis et cetera. Therefore, it is important to think of a possibility of adrenal infarction when faced with a scenario of abdominal pain during gestation, especially when the symptoms are sudden, severe and associated with nausea, vomiting or fever in the context of haemodynamic instability.

\section{Case Presentation}

25 year-old female, with her first pregnancy and 32 weeks into gestation, presented to the antenatal day unit with acute onset left flank pain. Initially thought to be musculoskeletal pain, the patient was discharged on simple analgesia. Later, the same day, in addition to the left flank pain, she developed sharp, central lower chest pain, back pain and associated vomiting. Pain score was 9/10. A few hours later, the patient developed right-sided abdominal pain. There was no associated fever or diarrhoea. There was no history of 
trauma. There was no preceding illness to report, and up to this point, the pregnancy had been progressing as per normal with a normal fetal anomaly scan a few months earlier. There was no past medical or surgical history of note nor was she taking any regular medication. There was no personal or family history of thromboembolic disease. She was employed as an assistant manager of a shop which did not require strenuous physical activity.

On clinical examination her initial blood pressure (BP) was 112/58 which later dropped to 100/64 with the heart rate (HR) $101-108$ beats/min. Her oxygen saturations were $100 \%$ on room air with a respiratory rate of 24-26 breaths/min. There was evidence of generalized mild abdominal tenderness.

She was noted to have a lactic acidosis of $9.0 \mathrm{mmol} / \mathrm{L}(0.5-1.0)$ with a bicarbonate of $13.8 \mathrm{mmol} / \mathrm{L}(23-28)$ on a venous blood gas (VBG) analysis. The electrocardiogram (ECG) did not show acute ischaemia or right heart strain pattern whilst the chest X-ray excluded pneumonia and air under diaphragm. Urine dip stick results did not suggest infection or significant protein loss but did show glucose and ketones $3+$ with blood 2+. Mid-stream urine culture was negative for organisms, and an ultrasound of the abdomen was unremarkable. She was reviewed by the Obstetrics team who thought this may be spontaneous rupture of membranes with septicaemia while the surgical team felt this could be a case of an acute abdomen i.e appendicitis/pancreatitis. Her initial blood test results (summarised in the table 1) and the clinical picture pointed to a diagnosis of intra-abdominal septicaemia in view of raised inflammatory markers, lactic acidosis and low bicarbonate levels. She was commenced on broad-spectrum intravenous antibiotics, intravenous fluids and analgesia.

Table 1: Initial blood test results

\begin{tabular}{|c|c|c|}
\hline Blood Test & Result & Normal \\
\hline Sodium & $134 \mathrm{mmol} / \mathrm{L}$ & $135-144$ \\
\hline Potassium & $4.1 \mathrm{mmol} / \mathrm{L}^{\prime}$ & $3.5-5.3$ \\
\hline Urea & $2.5 \mathrm{mmol} / \mathrm{L}$ & $3-7.6$ \\
\hline Creatinine & 48 umol/L & $55-87$ \\
\hline CRP & $38 \mathrm{mg} / \mathrm{L}$ & $0-8$ \\
\hline $\mathrm{PT}$ & $11.4 \mathrm{~s}$ & $10-13.5$ \\
\hline APTT & $25.9 \mathrm{~s}$ & $28-38$ \\
\hline Haemoglobin & $115 \mathrm{~g} / \mathrm{L}$ & $120-160$ \\
\hline WCC & $23.3 \times 10^{9} / \mathrm{L}$ & $4-11$ \\
\hline Platelets & $446 \times 10^{9} / \mathrm{L}$ & $150-400$ \\
\hline MCV & $80.9 \mathrm{fL}$ & $80-100$ \\
\hline $\mathrm{D}$ dimer & $673 \mathrm{ng} / \mathrm{mL}$ & $0-300$ \\
\hline Amylase & $32 \mathrm{U} / \mathrm{L}$ & $33-153$ \\
\hline $\mathrm{PH}$ & 7.28 & $7.35-7.45$ \\
\hline Bicarbonate & $13.8 \mathrm{mmol} / \mathrm{L}$ & $23-26$ \\
\hline Base Excess & $-13.3 \mathrm{mmol} / \mathrm{L}$ & +2 to -2 \\
\hline Lactate & $9.0 \mathrm{mmol} / \mathrm{L}$ & $0-1.3$ \\
\hline
\end{tabular}

Key:

C-reactive protein (CRP), Prothrombin Time (PT), Activated Partial Thromboplastin Time (APTT), White Cell Count (WCC), Mean Corpuscular Volume (MCV)

In view of unexplained severe symptoms, raised inflammatory markers and significant lactic acidosis, a contrast-enhanced CT scan of the abdomen and pelvis was done after discussing benefits and risks with the patient. This revealed an enlarged left adrenal gland with low attenuation in keeping with infarction (Figure 1a and Figure 1b). 
Figure 1(a):

Contrast-enhanced CT abdomen and pelvis in the axial view. The arrow shows an enlarged left adrenal gland with low attenuation when compared to the right.

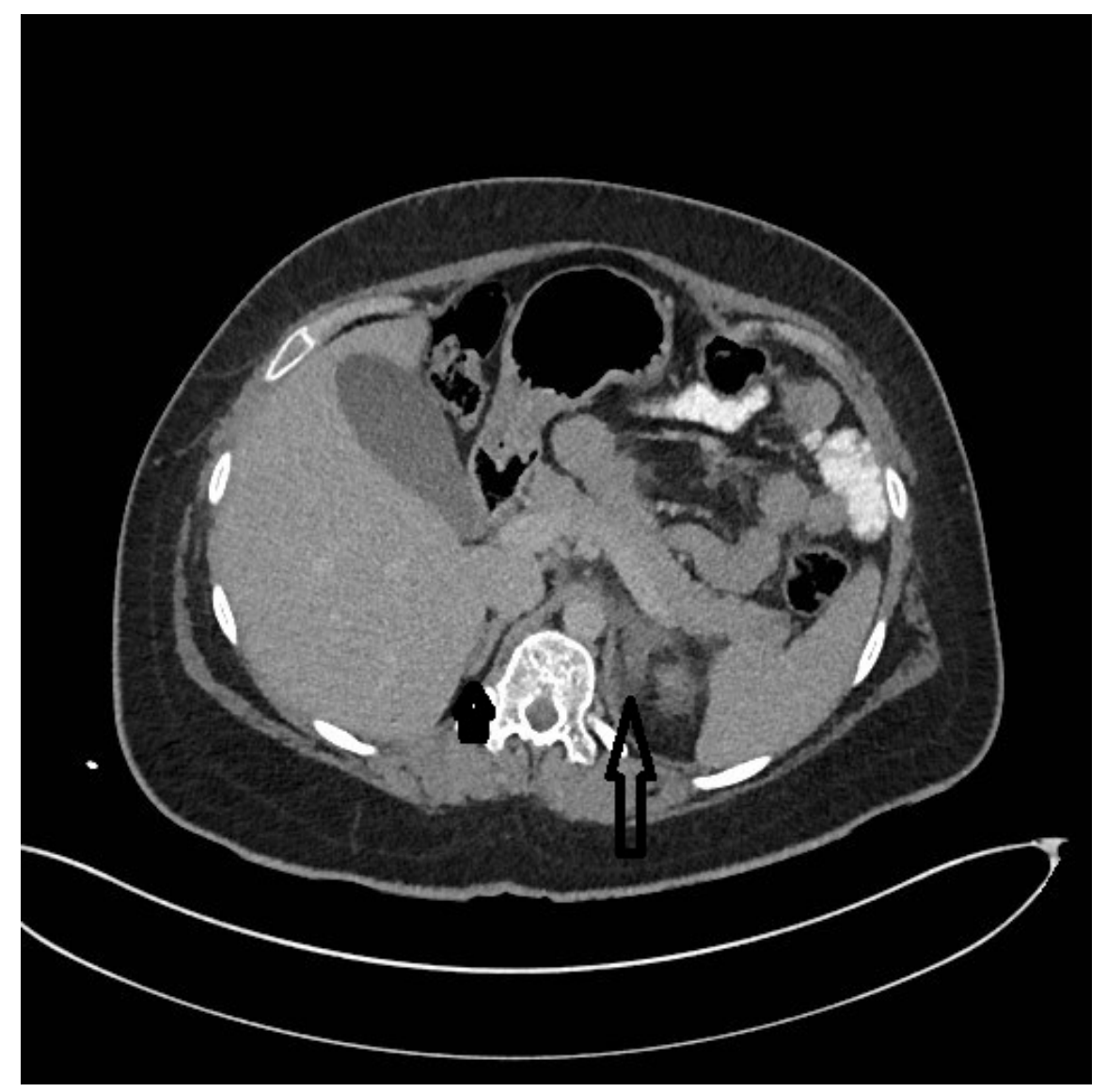

Figure 1(b):

Contrast enhanced CT abdomen and pelvis in the coronal view. The hollow black arrow depicts an enlarged left adrenal gland with low attenuation when compared to the right. 


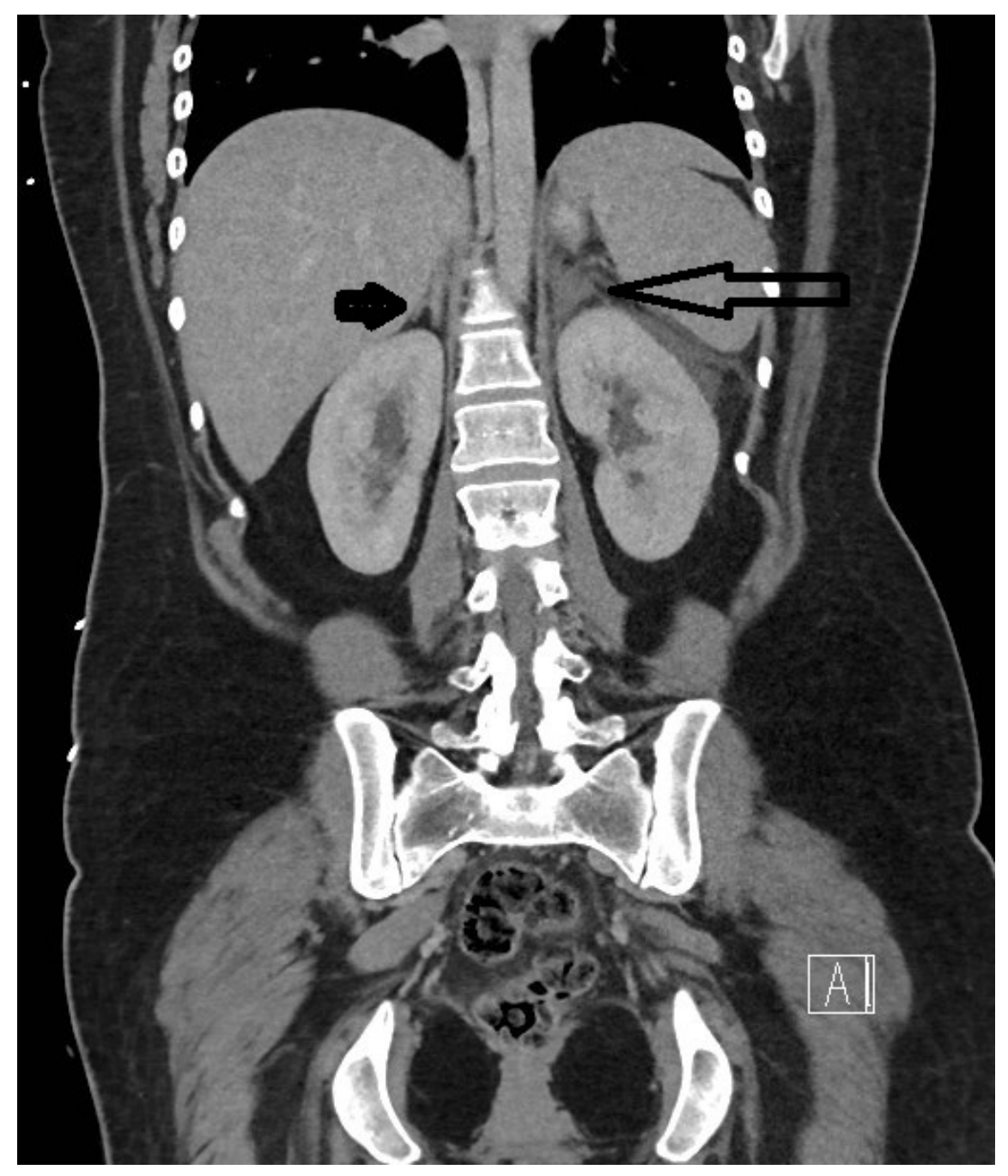

The following day random serum cortisol was done at 1410 hours which came back at 557nmol/L (200-700). The patient had spontaneous rupture of membranes, and a female baby weighing $2.2 \mathrm{lbs}$ was delivered by an emergency C-section who then required admission to the neonatal intensive care unit for breathing difficulties however, thankfully, did go on to make a full recovery. The mother continued to have abdominal and back pain and became hypotensive. Metabolic and lactic acidosis improved following conservative management. On day three, postpartum (0900 hours) serum cortisol was 78nmol/L (200-700). Bilateral adrenal infarction was suspected, and a dedicated contrast-enhanced adrenal CT scan was done which confirmed left adrenal infarction with low attenuation and oedema but the right adrenal gland was radiologically normal (Figure $2 \mathrm{a}$ and Figure 2b).

Figure 2(a):

Contrast-enhanced CT adrenal gland in the axial view. The arrow clearly shows an enlarged and oedematous left adrenal gland compared to the right. 


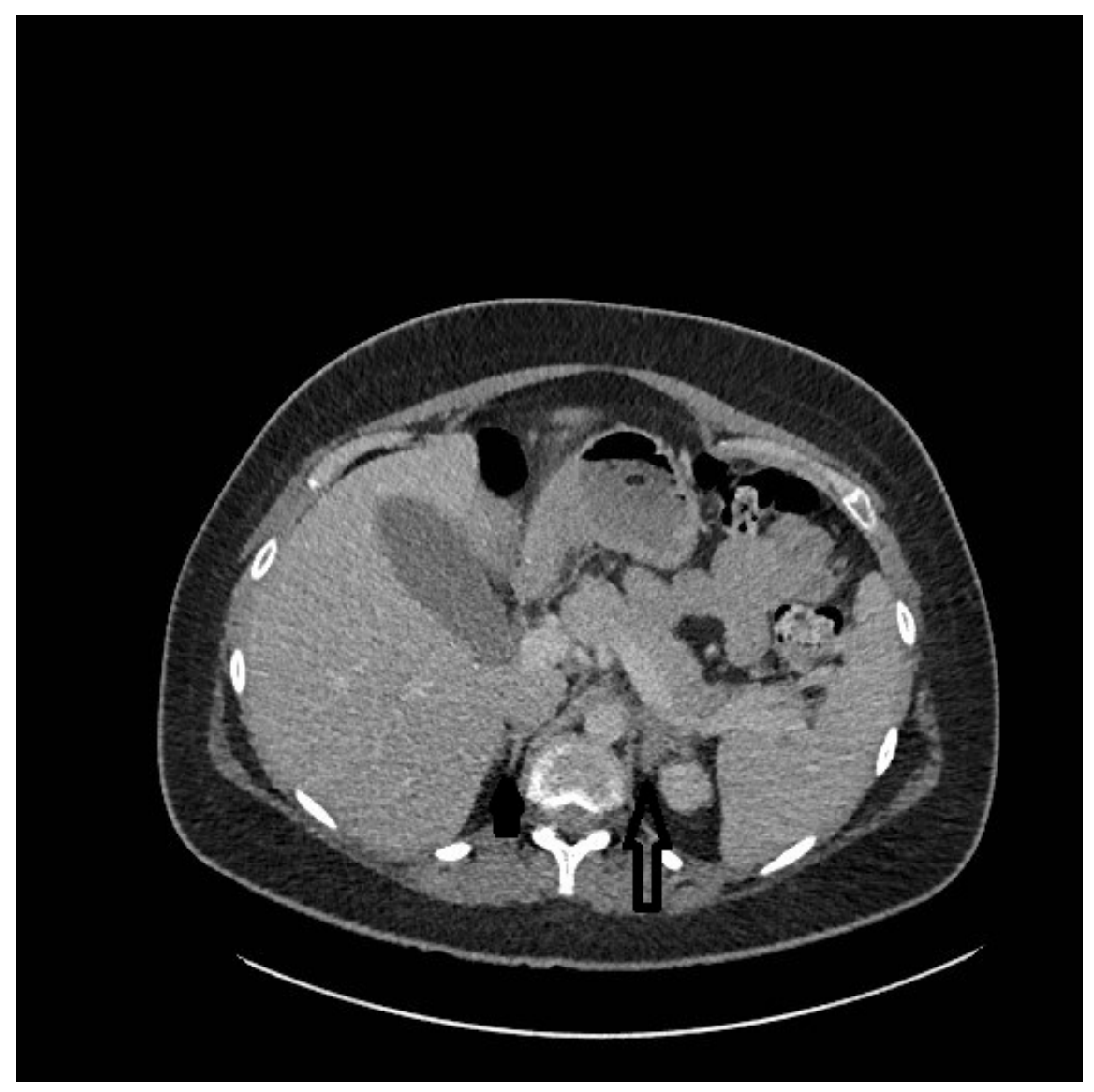

Figure 2(b):

Contrast-enhanced CT adrenal gland in the coronal view. An enlarged and oedematous left adrenal gland (hollow arrow) can clearly be visualised compared to the right.

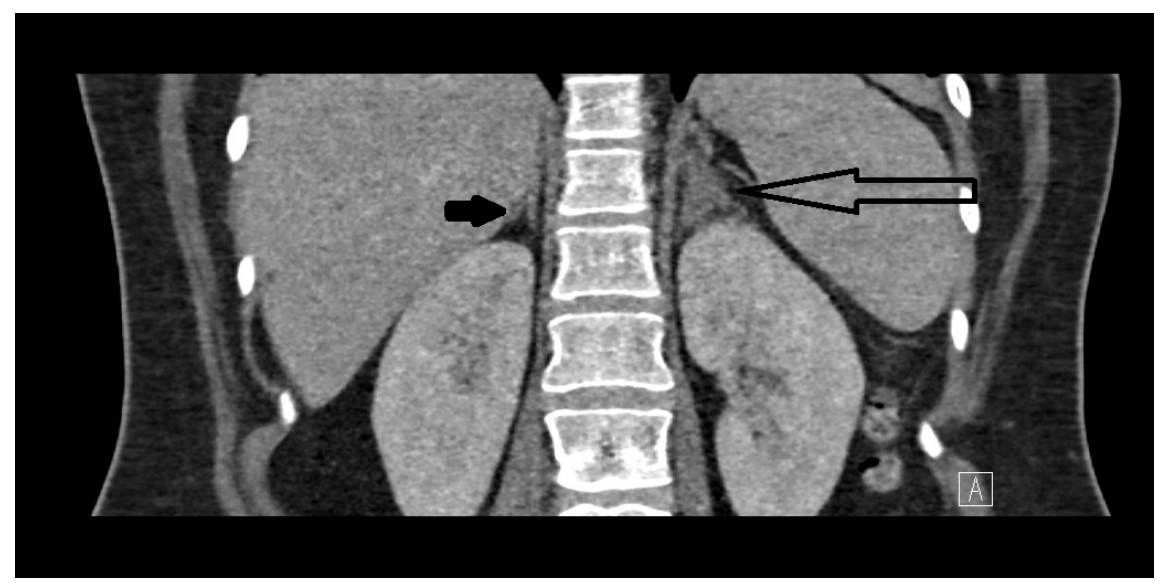

She was commenced on Hydrocortisone $20 \mathrm{mg}$ at 0800 hours and $10 \mathrm{mg}$ at 1400 hours and anticoagulated with warfarin in order to prevent progression of the infarction and contralateral thrombosis. Once her symptoms resolved, she was discharged with endocrinology follow up. Four months after the initial event, she was 
reviewed in the endocrinology clinic with a short synacthen test (SST) which revealed $\mathrm{T}^{0}$ cortisol $263 \mathrm{nmol} / \mathrm{L}$ and $\mathrm{T}^{30} 407 \mathrm{nmol} / \mathrm{L}$. She was maintained on hydrocortisone $20 \mathrm{mg}$ at 0800 hours and $10 \mathrm{mg}$ at 1400 hours whilst her anticoagulation in the form of warfarin was discontinued. Two months later, she was seen with an MRI of her abdomen, which showed complete resolution of the left adrenal infarction (Figure 3).

Figure 3:

MRI abdomen in the axial view showing a normal left adrenal gland.

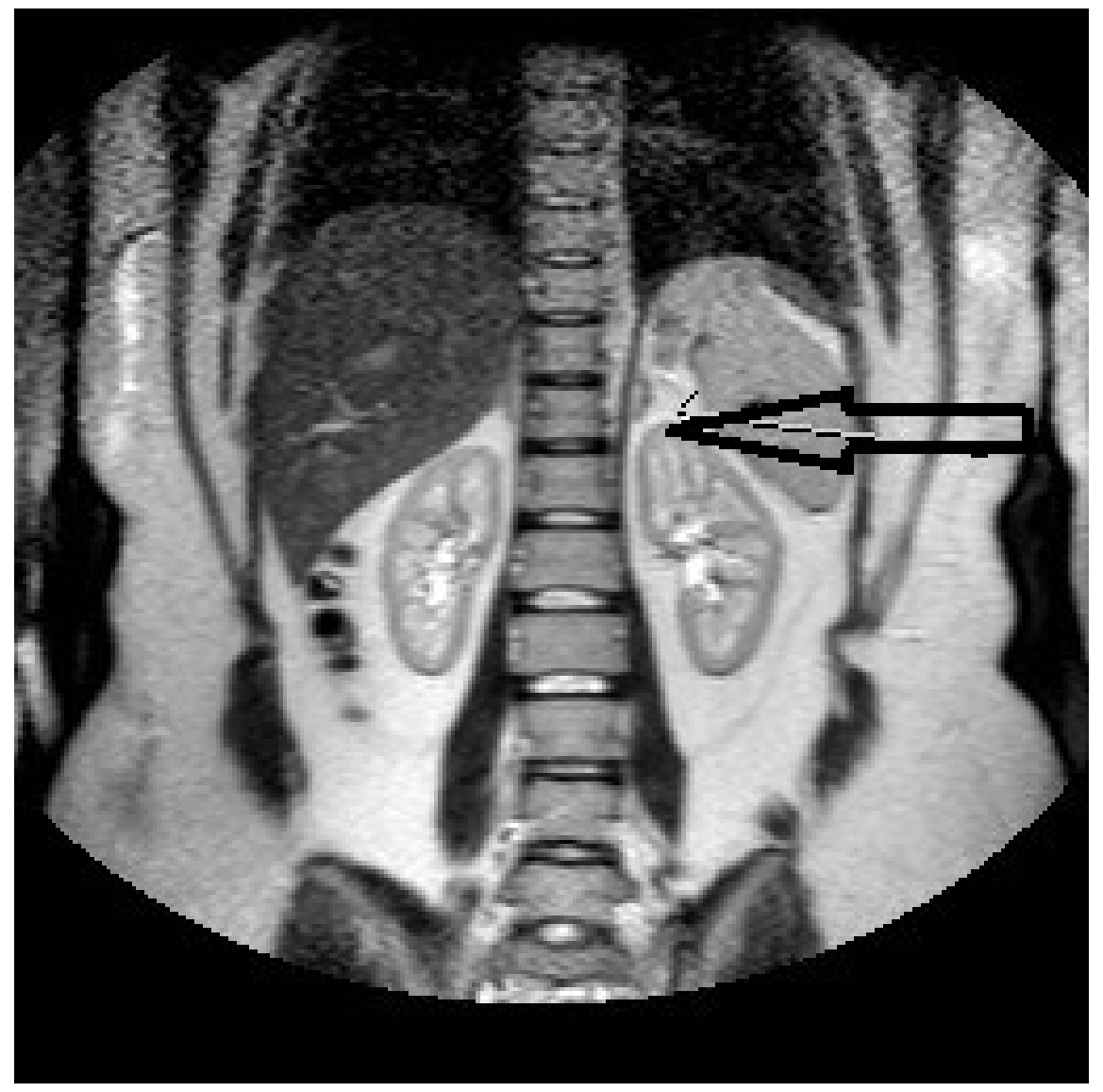

The dose of hydrocortisone was reduced to $10 \mathrm{mg}$ at 0800 hours and $5 \mathrm{mg}$ at 1400 hours. Three months later she was reviewed again with a repeat SST which showed $\mathrm{T}^{0}$ cortisol $150 \mathrm{nmol} / \mathrm{L}$ and $\mathrm{T}^{30}$ cortisol 585nmol/L. She was maintained on hydrocortisone $10 \mathrm{mg}$ at 0800 hours and $5 \mathrm{mg}$ at 1400 hours. Seven months later her steroid reserve had completely recovered with SST showing $\mathrm{T}^{0}$ cortisol $529 \mathrm{nmol} / \mathrm{l}$ and $\mathrm{T}^{30}$ cortisol $661 \mathrm{nmol} / \mathrm{L}$ (Table 2 summarises serial SST results). Hydrocortisone at this visit was discontinued, and the patient was discharged from follow up. Apart from pregnancy, no other thrombogenic cause was found. Prophylactic anticoagulation with low molecular weight heparin (LMWH) was recommended for the duration of any future pregnancies.

Table 2: Serial SST results

\begin{tabular}{lll}
\hline Time & SST - Cortisol (nmol/L) & Hydrocortisone dose $(\mathrm{mg})$ \\
\hline 4 months post event & $\mathrm{T}^{0} 263 \mathrm{~T}^{30} 407$ & $20 / 10$ \\
9 months post event & $\mathrm{T}^{0} 150 \mathrm{~T}^{30} 585$ & $10 / 5$
\end{tabular}




\begin{tabular}{lll}
\hline Time & SST - Cortisol $(\mathrm{nmol} / \mathrm{L})$ & Hydrocortisone dose $(\mathrm{mg})$ \\
\hline 16 months post event & $\mathrm{T}^{0} 529 \mathrm{~T}^{30} 661$ & Discontinued \\
\hline
\end{tabular}

\section{Discussion}

Adrenal infarction has been reported with hypercoagulable states including antiphospholipid syndrome ${ }^{1}$, myelodysplasia ${ }^{2}$, Crohn's disease ${ }^{3}$ and more recently, COVID-19 infection ${ }^{4}$ among other pathologies.

We present a case of unilateral adrenal infarction in pregnancy with a spontaneous biochemical and radiological resolution. Unilateral adrenal infarction in pregnancy is rare ${ }^{5-7}$ with a reported incidence of $1.3 \%$ on MRI scans at one institution done for abdominal pain ${ }^{8}$.

There are several predisposing factors for developing adrenal infarction in pregnancy. Pregnancy is a hypercoagulable state, which, from an evolutionary perspective, is a protective mechanism against bleeding due to miscarriage or during childbirth ${ }^{9}$. Studies have shown that pregnant women are at a $4-5$ fold increased risk of thromboembolism during pregnancy and the postpartum period compared with the non-pregnant state ${ }^{9}$. There are several reasons for this. Normal pregnancy is accompanied by increased concentration of factors VII, VIII, X, and von Willebrand factor and by pronounced increases in fibrinogen ${ }^{10}$. Free protein S, the active, unbound form, is decreased during pregnancy secondary to increased levels of its binding protein, the complement component $\mathrm{C}_{4} \mathrm{~b}^{10}$. All these increase the likelihood of non-haemorrhagic infarctions. This increased thrombogenic state is likely to affect the adrenal gland because of its unique vasculature with rich arterial supply but poor venous drainage.

Evaluation of acute abdominal pain in pregnancy is challenging because of an enlarged uterus, presence of varying volumes of amniotic fluid and change in the anatomy associated with pregnancy. The common non-obstetric differential diagnosis for acute abdominal pain in pregnancy ${ }^{11,12}$ that can be recognised with imaging are acute appendicitis, cholelithiasis, cholecystitis, pancreatitis, and bowel disease. However, adrenal infarction should be included in the differential diagnosis of severe upper abdominal pain in pregnancy ${ }^{5-7}$, especially if there is a persistent abdominal and flank pain, and the above mentioned common causes are ruled out. The diagnosis can be made by an adrenal CT, contrast-enhanced MRI or a non-contrast MRI scan. However, adrenal CT and contrast-enhanced MRI are generally avoided during pregnancy due to foetal radiation exposure ${ }^{13}$, and non-contrast MRI is the investigation of choice. There is no consensus about the sequence of SST and imaging in these situations. However, an SST can be done prior to imaging in high-risk patients with personal or family history of autoimmune disease, malignancyor in patients who refuse abdominal scanning. In our patient, the SST clearly demonstrated adrenal insufficiency, and she was started on steroid replacement. Interestingly, the right adrenal gland was radiologically completely normal on the CT scan; however, it did not compensate for the adrenal insufficiency. It is possible that the changes in the right adrenal gland were subtle and not visualised on the initial CT scan.

There is no consensus on the follow-up imaging and reassessment of adrenal reserves in this group of patient. In our patient, we repeated an MRI scan and SST approximately six months after the first scan. Interestingly, her MRI scan at follow-up showed complete resolution of the infarction, and the SST confirmed biochemical resolution of the adrenal insufficiency. There are very few reports in the literature documenting the spontaneous resolution of pregnancy-related adrenal infarcts, and our report highlights the importance of repeat scanning and repeat SST in this group of patients.

In the setting of adrenal infarction, the patient was anticoagulated to prevent contralateral thrombosis. Warfarin was chosen to anticoagulate the patient as she had already delivered her child. Use of coumarin derivatives by the mother at any time during pregnancy may increase the risk of central nervous system anomalies in the foetus ${ }^{14}$. There is also no consensus on the duration of anticoagulation in such cases at present. It is reasonable to consider unilateral adrenal infarction in pregnancy as a provoked venous thromboembolism event in the absence of thrombogenic disease and as such three to six months of anticoagulation is a reasonable approach. 
This case highlights that it may take up to 6 months before restoration of the adrenocortical steroid reserve, and thus patients should be followed up with SST's under endocrinology for this time period at least. Once Unilateral NHAI is confirmed, patients should be offered anticoagulation to prevent progression of infarction and contralateral thrombosis. Benefits vs risks of anticoagulation should always be considered, and where possible, patients should always be included in the decision-making process. More studies are required in order to ascertain a safe and effective duration of anticoagulation in such cases. In patients who develop adrenal infarction during gestation; we recommend prophylactic anticoagulation for the duration of subsequent pregnancies. After the acute event, follow up adrenal imaging should be considered as patients may develop adrenal haemorrhage due to revascularisation or atrophy of the gland. Follow-up imaging will also help to assess recovery of the adrenal gland, which may take up to 6 months, as described by our case.

\section{Conclusion}

Increased concentration of endogenous procoagulants and venous stasis increased the risk of adrenal infarction in pregnancy. This case asserts the importance of screening for adrenal insufficiency in pregnant women presenting with abdominal pain, nausea and haemodynamic instability.

\section{Author list and contribution}

Author 1: Najeeb Shah

First and the corresponding author who conceptualized the project, obtained informed written consent, wrote the first draft and all subsequent revisions of the manuscript.

Author 2: Harshal Deshmukh

Assisted with the drafting of the manuscript, undertook the literature search, and made constructive comments to improve the quality of the manuscript.

Author 3: MJ Akbar

Obtained all the suitable radiological images and made comments to improve the quality of the manuscript.

Author 4: Yamna Saeed

Made comments to improve the quality of the manuscript and edited the final draft.

Author 5: Shah Malik

Made comments to improve the quality of the manuscript and edited the final draft.

Author 6: Belinda Allan

Conceptualized the project, made comments to improve the quality of the manuscript, and provided overall supervision of the project as the senior author.

\section{Data Availability}

As this is a case report, anonymised data can be made available by the authors upon reasonable request subject to local confidentiality and ethics guidelines.

\section{Conflict of Interest}

There are no conflicts of interest to declare from any author with regards to this publication.

\section{Funding Statement}

This work received no funding.

\section{Ethics approval}

Not required. 


\section{Consent Statement}

Written informed consent was obtained from the patient for the publication of this case report and associated images.

\section{Acknowledgements}

I would like to acknowledge Dr Ewan Masson, Diabetes \& Endocrinology Consultant (retired) Hull University Teaching Hospitals NHS Trust for his encouragement and guidance in writing up this case.

\section{References:}

1. Espinosa G, Santos E, Cervera R, et al. Adrenal involvement in the antiphospholipid syndrome: clinical and immunologic characteristics of 86 patients. Medicine 2003; 82: 106-118.

2. Lockett HA, Hamilton-Wood C and Vaidya B. Addison's disease due to bilateral adrenal infarction in a patient with myelodysplastic syndrome.Eur J Gen Med 2011; 8: 72-74.

3. Khandelwal A, Krishna JS, Khandelwal K, et al. Bilateral adrenal infarction in Crohn's disease. Indian journal of endocrinology and metabolism 2013; 17: 933.

4. Leyendecker P, Ritter S, Riou M, et al. Acute adrenal infarction as an incidental CT finding and a potential prognosis factor in severe SARS-CoV-2 infection: a retrospective cohort analysis on 219 patients.European radiology 2021; 31: 895-900.

5. Aljenaee KY, Ali SA, Cheah SK, et al. Unilateral adrenal infarction in pregnancy secondary to elevated factor VIII. Saudi Med J 2017; 38: 654-656. DOI: 10.15537/smj.2017.6.18520.

6. Chasseloup F, Bourcigaux N and Christin-Maitre S. Unilateral nonhaemorrhagic adrenal infarction as a cause of abdominal pain during pregnancy. Gynecol Endocrinol 2019; 35: 941-944. DOI: 10.1080/09513590.2019.1622088.

7. Green PA, Ngai IM, Lee TT, et al. Unilateral adrenal infarction in pregnancy. BMJ Case Rep 2013; 2013. DOI: $10.1136 /$ bcr-2013-009997.

8. Glomski SA, Guenette JP, Landman W, et al. Acute Nonhemorrhagic Adrenal Infarction in Pregnancy: 10-Year MRI Incidence and Patient Outcomes at a Single Institution. AJR Am J Roentgenol 2018; 210: 785-791. 2018/02/16. DOI: 10.2214/ajr.17.18739.

9. Bremme KA. Haemostatic changes in pregnancy. Best Pract Res Clin Haematol 2003; 16: 153-168. DOI: 10.1016/s1521-6926(03)00021-5.

10. James AH. Pregnancy-associated thrombosis. Hematology Am Soc Hematol Educ Program 2009: 277285. DOI: 10.1182/asheducation-2009.1.277.

11. Bouyou J, Gaujoux S, Marcellin L, et al. Abdominal emergencies during pregnancy. J Visc Surg 2015; 152: S105-115. DOI: 10.1016/j.jviscsurg.2015.09.017.

12. Cappell MS and Friedel D. Abdominal pain during pregnancy.Gastroenterol Clin North Am 2003; 32: 1-58. DOI: $10.1016 / \mathrm{s} 0889-8553(02) 00064-\mathrm{x}$.

13. Committee on Obstetric P. Committee Opinion No. 723: Guidelines for Diagnostic Imaging During Pregnancy and Lactation. Obstet Gynecol2017; 130: e210-e216. DOI: 10.1097/AOG.0000000000002355.

14. Pati S and Helmbrecht GD. Congenital schizencephaly associated with in utero warfarin exposure. Reprod Toxicol 1994; 8: 115-120. DOI: 10.1016/0890-6238(94)90018-3. 